

\title{
Metallicities in stars - what solar neutrinos can do
}

\author{
Kai Zuber* \\ Institute for Nuclear and Particle Physics, Technical University Dresden, 01069 Dresden, \\ Germany \\ E-mail: zuber@physik.tu-dresden.de
}

\begin{abstract}
New elemental abundance determinations from the solar photosphere have spoiled the extremely good agreement of helioseismological data and solar models. One of the fundamental assumptions of stellar evolution, namely the homogeneous distribution of metals in stars, in on the bench. Probably the only way to probe the metal abundance in the Interior of the Sun is the observation of $\mathrm{CNO}$ neutrinos. The current status and future potential of solar neutrino experiments to do so is discussed.
\end{abstract}

XII International Symposium on Nuclei in the Cosmos,

August 5-12, 2012

Cairns, Australia

${ }^{*}$ Speaker. 


\section{Introduction}

Detailed understanding of solar processes is a fundamental ingredient for stellar astrophysics and stellar modeling. The occurrence of the solar neutrino problem caused more intense simulations of solar models (called Standard Solar Models, SSM) and the temperature dependence of the individual flux contributions. The solution of the problem of missing solar neutrinos by SuperKamiokande, the gallium experiments GALLEX and SAGE but especially SNO had major impact on neutrino physics and showed that our basic understanding of energy production in the Sun is correct. The measurement of the total ${ }^{8} \mathrm{~B}$ flux of SNO was the first time in solar neutrino physics that experimental errors were smaller than the flux prediction uncertainties. This is accompanied by cross-section measurements of fundamental reactions within the Sun especially by the LUNA experiment in the Gran Sasso Underground Laboratory (see [1] for a new compilation of S-factors of solar reactions). Finally, the major progress of helioseismology measuring the speed of sound and thus pressure and density profiles within the Sun to tremendous precision. Currently this is very successfully expanding into astroseismology. The agreement of solar model predictions and helioseismological measurements was extraordinarily good about a decade ago. Recently, a more sophisticated 3D-fit to photospheric spectral lines led to a new abundance determination of the Sun. One major result is the reduction of the $\mathrm{C}, \mathrm{N}, \mathrm{O}, \mathrm{Ne}$ by about 30-40\%, however the robustness of the deduced abundancies is still discussed [2]. This is part of the reduction of metals in general, which is imposed on solar models as input parameter. The effect of the new abundancies in the solar models with respect to helioseismology is shown in Fig. 1. Obviously the agreement with observation is worse than before. The same is true for the lower edge of the convection zone and the surface helium abundance. This is now called "the solar abundance" problem. For recent reviews on this issue see $[3,6,7]$.

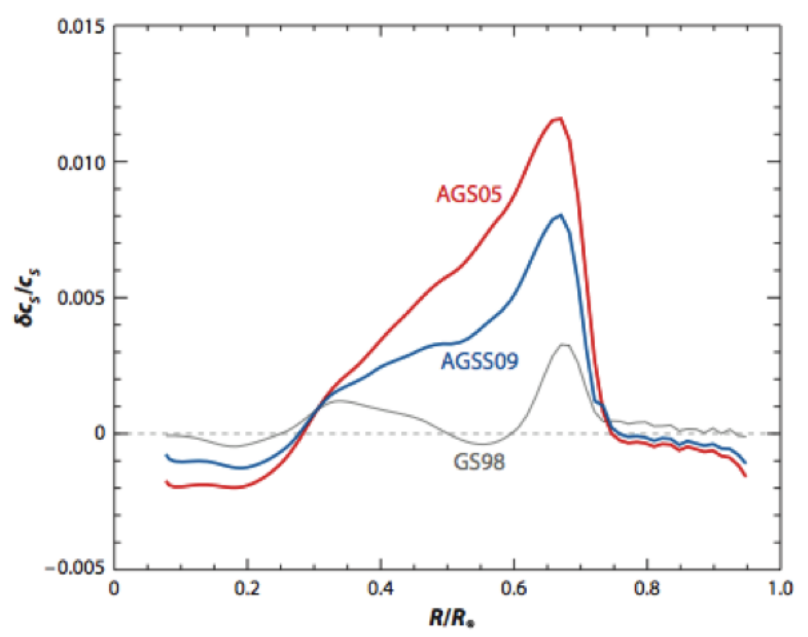

Figure 1: Relative sound speed change as a function of solar radius for previous abundances (grey) [4], the first new determination (red) [5] and the most recent compilation (blue) [3]. Evidently the abundance determination in 2005 led to the worst agreement (from [3]).

Furthermore in solar modeling there is a degeneracy between opacity and elemental abundancies. Helioseismology has only little sensitivity of light elements like C,N as their effect on opacity 

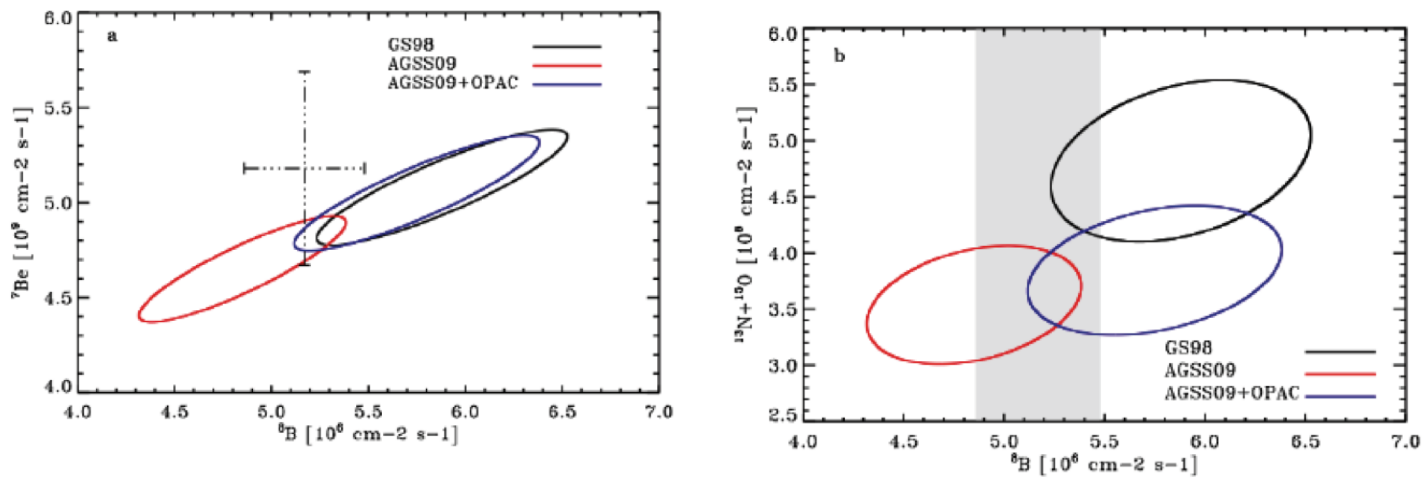

Figure 2: Shown are the expected fluxes from ${ }^{7} \mathrm{Be}$ and ${ }^{8} \mathrm{~B}$ (left) and the ones from ${ }^{13} \mathrm{~N}$ and ${ }^{15} \mathrm{O}$ versus ${ }^{8} \mathrm{~B}$ (right). As can be seen, current data cannot discriminate among the different solutions. However, a ${ }^{13} \mathrm{C}$ measurement would help to remove the degeneracy between opacity and elemental abundance. (from [8]).

is dominantly in the convective envelope, elements like $\mathrm{O}$ and $\mathrm{N}$ have a major impact around the lower edge of the convective zone while the heavy elements dominate the opacity in the core region. Hence, changing opacities could help to improve the agreement of models with helioseismological observation again. Also ${ }^{7} \mathrm{Be}$ and ${ }^{8} \mathrm{~B}$ flux measurements cannot discriminate between the two abundance options as shown in Fig. 2. However a ${ }^{13} \mathrm{~N}$ neutrino measurement would break the mentioned degeneracy as the ${ }^{13} \mathrm{~N}$ flux is correlated with the ${ }^{8} \mathrm{~B}$ flux, which is shown as well.

\section{CNO-neutrinos}

Probably the only chance to determine the metal content within the Sun is an observation of solar CNO-neutrinos. The contribution of the CNO-cycle to solar energy production is less than a percent, nevertheless there is a reasonable solar neutrino flux (around $5 \times 10^{8} \mathrm{~cm}^{-2} \mathrm{~s}^{-1}$ on Earth) as shown in Fig. 3. As can be seen the interesting ${ }^{13} \mathrm{~N}$ and ${ }^{15} \mathrm{O}$ components have endpoints at 1.2 and $1.73 \mathrm{MeV}$ respectively and thus are below the threshold of Cherenkov-detectors.

\section{Current limits}

Currently CNO-neutrinos can only be measured in neutrino-electron scattering experiments. The only one running at the moment is Borexino, a 300 ton liquid scintillator experiment in the Gran Sasso Laboratory. The observable is the electron recoil due to scattering. The obtained spectrum of Borexino so far is shown in Fig. 4. Major features interfering with the CNO spectrum are electrons from ${ }^{7} \mathrm{Be}$ and decays from ${ }^{85} \mathrm{Kr},{ }^{210} \mathrm{Bi}$ and ${ }^{11} \mathrm{C}$. As can be seen the expected $\mathrm{CNO}$-spectrum is very similar to the ${ }^{210} \mathrm{Bi}$ decay spectrum. Hence, only an upper limit of 7.7 $\times 10^{8} \mathrm{~cm}^{-2} \mathrm{~s}^{-1}(95 \% \mathrm{CL})$ can be given assuming the LMA solution for $v_{e}$ oscillations. This is still higher than all predicted model fluxes. The solar luminosity constraint, the fact that the rate of the pp- and CNO cycle weighted by energy of the individual chains deposited the Sun, is fixed by the photon luminosity

$$
\frac{L_{\odot}}{4 \pi(A . U .)^{2}}=\sum_{i} \alpha_{i} \Phi_{i}
$$






Figure 3: The solar neutrino spectrum. The largest component is due to the fundamental pp-reaction, the highest energetic components are the ${ }^{8} \mathrm{~B}$ and hep-flux. All neutrinos fluxes from the CNO-cycle are plotted in red (from [9]).

whith $\Phi_{i}$ as the individual solar neutrino fluxes and $\alpha_{i}$ has to be calculated from the SSM, allows another estimate. Hence, there is a correlation between the pp-flux and the CNO-flux which is shown using the measured fluxes as in Fig. 4.
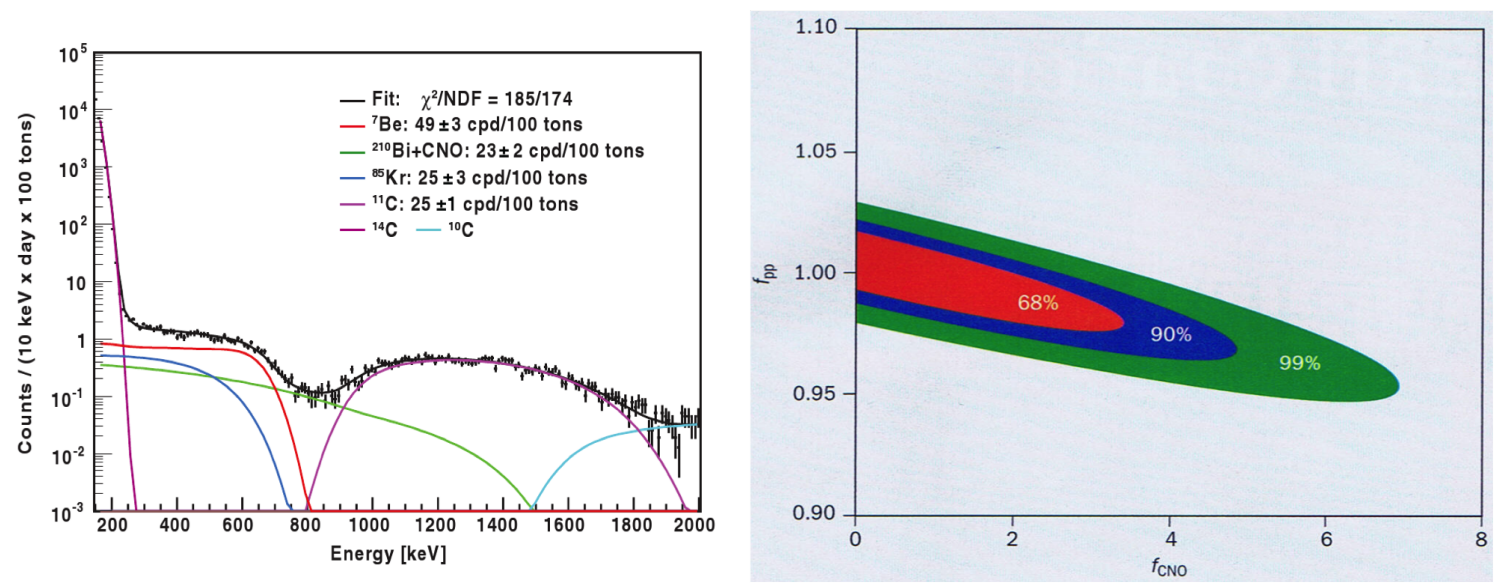

Figure 4: Left: Measured energy spectrum of the Borexino experiment. Shown are the fitted individual components. A common fit of ${ }^{210} \mathrm{Bi}$ and $\mathrm{CNO}$ neutrinos already indicates that both spectra look experimentally very similar (from [10]). Right: pp-flux and CNO-flux in units of the SSM prediction. Using the correlation between the solar pp-flux and the CNO-flux, fixed by solar luminosity constraint together with the measured fluxes from Borexino a limit on the CNO-flux can be given.

\section{Future considerations}

How can the situation be improved? First of all Borexino made a major purification campaign on their detector and they are back for data taking with reduced background. New data will lead 
to improved numbers in the near future. A major issue remaining is the ${ }^{11} \mathrm{C}$ production by cosmic ray muons. This can be experimentally removed by a triple coincidence (the reaction of a muon on ${ }^{12} \mathrm{C}$ will produce a muon signal in addition to a neutron capture and the ${ }^{11} \mathrm{C}$ decay), however on the expense of reduced fiducial volume and enhanced dead times. Hence, going deeper would be preferential. In this way the planned SNO+ experiment, a 780 ton liquid scintillation detector using the former infrastructure of the SNO experiment at SNOLAB is beneficial. Due to the larger depth a reduction of a factor 100 is expected and furthermore the total volume is about 2.5 times larger than Borexino (Fig. 5). Independent of depth, a very low level in ${ }^{210} \mathrm{Bi}$ is necessary as its beta decay spectrum is very similar to the electron recoil spectrum produced by $\mathrm{CNO}$ neutrinos. A potential way to improve here is discussed in [11]. Unfortunately SNO+ has decided to do a low energy solar measurement only in a second phase of the experiment and thus postponed new results for several years. What other future very large scale experiments like LENA can do remains to be seen.


Figure 5: Schematic sketch of the ${ }^{11} \mathrm{C}$ background (grey) and the CNO-flux (red) in Borexino (left) and SNO+ (right) (Courtesy B. von Krosigk).

\section{References}

[1] E. G. Adelberger et al., Solar fusion cross sections II: the pp chain and CNO cycles, Rev. Mod. Phys.2011 (83) 135 [arXiv: 1004.2318$].$

[2] E. Caffau et al., The solar photospheric abundance of carbon. Analysis of atomic carbon lines with the CO5BOLD solar model, Astron. Astrophys. 2010 (514) A92 [arXiv: 1002 .2628].

[3] M. Asplund et al. , The chemical composition of the Sun, Ann. Rev. Astro. Astrophys. 2009 (47) 481

[4] N. Grevesse, A. J. Sauval, Standard Solar Composition, Space Sci. Rev. 1998 (85) 161

[5] M. Asplund, N. Grevesse, A. J. Sauval, in Astronomical Society of the Pacific Conference Series, Vol. 336, Cosmic Abundances as Records of Stellar Evolution and Nucleosynthesis, ed. T. G. Barnes, III \& F. N. Bash, p. 25, 2005

[6] W.C. Haxton, R. G. H. Robertson and A. Serenelli, Solar Neutrinos: Status and Prospects, [arXiv:1208.5723].

[7] V. Antonelli et al., Solar Neutrinos, [arXiv: 1208.1356]. 
[8] A. Serenelli, New Results on Standard Solar Models, Astroph. Space Sci. 2010 (38) 13 [arXiv:0910.3690].

[9] L. Stonehill, J. Formaggio and R. G. H. Robertson, Solar neutrinos from CNO electron capture, Phys. Rev. C 2004 (69) 015801 [hep-ph/ 0309266 ].

[10] C. Arpessella et al., New results on solar neutrino fluxes from 192 days of Borexino data, Phys. Rev. Lett. 2008 (101) 091302 [arXiv: 0805.3843 ].

[11] F. L. Villante et al., A step toward CNO solar neutrinos detection in liquid scintillators, Phys. Lett. B 2011 (701) 336 [arXiv: 1104 . 1335]. 